Our thanks to all those who have helped with this issue of Bioanalysis. Listed below are authors, referees and others who have kindly given their time, effort and expertise; their generosity has helped establish this publication.

$\begin{array}{llll}\text { Acharya V } & \text { Goto-Inoue N } & \text { Pawliszyn J } & \text { Tabler E } \\ \text { Agoram B } & \text { Hay L } & \text { Peoples M } & \text { Teale P } \\ \text { Arnold M } & \text { Hotha K } & \text { Phillips K } & \text { Termopoli V } \\ \text { Aubry A } & \text { Huang P } & \text { Pottgiesser T } & \text { Thorpe R } \\ \text { Barbas C } & \text { Jensen B } & \text { Pruvost A } & \text { Thway T } \\ \text { Bharathi V } & \text { Jiang Y } & \text { Rambla-Alegre M } & \text { Tracey R } \\ \text { Cao Z } & \text { Kaczmarek A } & \text { Remane D } & \text { Urbanowicz B } \\ \text { Carda-Broch S } & \text { Kaliszan R } & \text { Rojo D } & \text { van der Graaf P } \\ \text { Chatki K P } & \text { Liang Z } & \text { Rupasinghe M } & \text { Venkateswarlu S } \\ \text { Cheng X } & \text { Liu A } & \text { Rupérez F } & \text { Vuckovic D } \\ \text { Coats V } & \text { Lu Y } & \text { Samanidou V } & \text { Wang F } \\ \text { Dawes M } & \text { Ma M } & \text { Schug K } & \text { Waszczuk-Jankowska M } \\ \text { Deventer K } & \text { Ma Y } & \text { Schumacher O } & \text { Wen X } \\ \text { Dong H } & \text { MacNeill R } & \text { Schuster G } & \text { Yao M } \\ \text { Edlund P } & \text { Markuszewski M } & \text { Schwudke D } & \text { Ye Z } \\ \text { Farside A } & \text { Markuszewski M } & \text { Shaikh M } & \text { Yuan Y } \\ \text { Furtado M } & \text { McCurdy D } & \text { Shi Z } & \text { Zeng S } \\ \text { Gamagedara S } & \text { Moerkeberg J } & \text { Spriggs F } & \text { Zhao Y } \\ \text { Gao J } & \text { Moussallie M } & \text { Stomeyer R } & \text { Zou J } \\ \text { Geiser L } & \text { Myler H } & \text { Stouffer B } & \\ \text { Góra R } & \text { Palma P } & \text { Szumski M } & \end{array}$

\title{
INDEX OF ADVERTISERS
}

\section{Company}

Advertise with Bioanalysis

Bioanalysis zone

Subscribe to Bioanalysis

Bioanalysis Trial

Pharmaceutical Patent Analyst

Biomarkers in Medicine

Expert Review of Molecular Diagnostics

Personalized Medicine

Pharmacogenomics

Clinical Investigation

Future Medicinal Chemistry

Therapeutic Delivery

Waters
Page

Inside Front Cover 